\title{
Communication
}

\section{Facile In Situ Growth of Zif-8 Nanosheets with Enhanced Anti-Corrosion Performance for Carbon Steel in Seawater}

\author{
Zhishu Zhang ${ }^{1}$, Qingjian Liu ${ }^{2,3, *}$ and Gaocan Qi ${ }^{4}$ \\ 1 China National Offshore Oil Corporation (CNOOC) China Ltd., Zhanjiang 524057, China; \\ zhangzhsh@cnooc.com.cn \\ 2 Tianjin Key Laboratory for Advanced Mechatronic System Design and Intelligent Control, \\ School of Mechanical Engineering, Tianjin University of Technology, Tianjin 300384, China \\ 3 National Demonstration Center for Experimental Mechanical and Electrical Engineering Education, \\ Tianjin University of Technology, Tianjin 300384, China \\ 4 Tianjin Key Lab for Photoelectric Materials \& Devices, School of Materials Science and Engineering, \\ Tianjin University of Technology, Tianjin 300384, China; gaocanqi@tjut.edu.cn \\ * Correspondence: qingjian_liu_19840@163.com
}

check for updates

Citation: Zhang, Z.; Liu, Q.; Qi, G. Facile In Situ Growth of Zif-8 Nanosheets with Enhanced Anti-Corrosion Performance for Carbon Steel in Seawater. Coatings 2022, 12, 318. https://doi.org/ 10.3390/coatings12030318

Academic Editor: Shih-Chen Shi

Received: 10 January 2022

Accepted: 25 February 2022

Published: 28 February 2022

Publisher's Note: MDPI stays neutral with regard to jurisdictional claims in published maps and institutional affiliations.

Copyright: (C) 2022 by the authors. Licensee MDPI, Basel, Switzerland. This article is an open access article distributed under the terms and conditions of the Creative Commons Attribution (CC BY) license (https:// creativecommons.org/licenses/by/ $4.0 /)$.

\begin{abstract}
Carbon steel is one of the most important and widely used structural materials, but preventing its corrosion remains a great challenge. Herein, a metal-organic framework film consisting of Zif-8 nanosheets array was prepared using a one-step in situ growth method. This coating film can effectively inhibit the corrosion behavior of low carbon steel in seawater, resulting in improved corrosion resistance $\left(4010 \Omega \cdot \mathrm{cm}^{-2}\right)$ and low corrosion current density $\left(23 \mu \mathrm{A} \cdot \mathrm{cm}^{-2}\right)$. After long-term immersion in seawater, no notable pitting corrosion was observed and the film integrity was well preserved, demonstrating the feasibility of Zif-8 film for anti-corrosion coating.
\end{abstract}

Keywords: low carbon steel; anti-corrosion coating; metal-organic framework film; in situ growth; seawater medium

\section{Introduction}

As one of the most valuable industrial products, carbon steel-based materials have been used broadly in marine environments, such as in bridges, pipelines and ships [1-3]. However, the corrosion behavior of carbon steel in seawater not only threatens public safety, but also induces huge economic losses and environmental pollution [4]. Thus, the development of an effective corrosion-protection strategy to decrease the corrosion rate of carbon steel has become an urgent challenge.

Currently, the introduction of a passivation layer on the surface of carbon steel is one of the most promising approaches used to improve the corrosion resistance of carbon steel [1,4], which can effectively avoid its direct contact with a corrosive medium. For example, metal oxide films [5], layered double hydroxides [6], and organic silica coatings [7] have been designed as the passivation layers for carbon steel reinforcements [8]. It should be noted that most of these studies have focused on the corrosion behavior in $\mathrm{NaCl}$ or $\mathrm{HCl}$ solutions, whereas related investigations in seawater are rarely reported. Besides, traditional inorganic passivation layers typically show environmental toxicity while most of the reported organic passivation layers have insufficient anti-corrosion ability in neutral media. Thus, it is desirable to develop effective and environmentally acceptable corrosion inhibitors.

Metal-organic frameworks (MOFs) [9], which are a type of organic-inorganic hybrid material, have received considerable interest due to their morphological controllability, good chemical stability, large surface area, low density, and high crystallinity. Based on these promising properties, MOFs have been used in various fields, such as gas separation [10], drug delivery [11], enzyme carriers [12], catalysis [13], batteries [14], etc. Besides, there are some reports in the literature about the application of MOFs (such as Zif-8, MIL-53 
and $\mathrm{UiO}-66)$ in the field of corrosion protection for metals or alloys [14]. Nevertheless, to the best of our knowledge, the corrosion-protection performance of MOFs in seawater has not been reported. Meanwhile, the poor adhesion to c onquer the continuous invasion of corrosive species is still not fully solved. In this regard, the in situ growth of Zif- 8 nanosheets array on carbon steel is demonstrated to efficiently prevent the penetration of water and halide ions into the surface of carbon steel. The anti-corrosion performance was evaluated by polarization curves, electrochemical impedance spectroscopy, and a long-term immersion test. The present work provides a new approach to enhance the application of MOFs-based materials in metal-corrosion protection.

\section{Experimental Section}

Synthesis: In a typical procedure, $0.6 \mathrm{~g}$ of zinc nitrate hexahydrate and $1.3 \mathrm{~g}$ of 2-methylimidazole were separately dissolved in $30 \mathrm{~mL}$ deionized water and stirred for $30 \mathrm{~min}$. Then, the zinc nitrate hexahydrate solution was added into the 2-methylimidazole solution. After that, a piece of carbon steel $\left(10 B 21,2 \mathrm{~cm}^{2}\right)$ was immersed into the mixed solution and kept still for $6 \mathrm{~h}$. Subsequently, the carbon steel was washed with deionized water and dried in an oven at $60^{\circ} \mathrm{C}$ for $24 \mathrm{~h}$.

Characterization: The crystallographic phase of the prepared sample was analyzed by X-ray diffraction (XRD, D8 Advance) (Brooke, Karlsruhe, Germany) and Fourier transform infrared spectrometry (FT-IR) (Nicolet 6700, Thermo Scientific, Waltham, MA, USA). The ATR mode was used to acquire the FT-IR spectrum. The morphologies were observed by scanning electron microscopy (SEM, S4800, Hitachi, Tokyo, Japan) and transmission electron microscopy (TEM, JEM-2100F, JEOL, Tokyo, Japan). A dual-beam focused ion beam (FIB) system (Helios 650, FEI, Hillsboro, OR, USA) was used to prepare the sample for scanning transmission electron microscopy (STEM, Themis G2, FEI, Hillsboro, OR, USA) characterization. Electrochemical measurements were conducted in seawater (Bohai, Tianjin, China). Pt rod and Ag/AgCl electrodes were employed as the counter electrode and reference electrode, respectively. Electrochemical impedance spectroscopy (EIS) measurements were conducted in the frequency range of $1 \mathrm{MHz}$ to $0.1 \mathrm{~Hz}$. According to methods described in the literature [15], artificial seawater was used as the electrolyte, and the $\mathrm{pH}$ was set to 8.2. The chemical composition of artificial seawater was $26.5 \mathrm{~g} \mathrm{~L}^{-1} \mathrm{NaCl}$, $24 \mathrm{~g} \mathrm{~L}^{-1} \mathrm{MgCl}_{2}, 0.73 \mathrm{~g} \mathrm{~L}^{-1} \mathrm{KCl}, 3.3 \mathrm{~g} \mathrm{~L}^{-1} \mathrm{MgSO}_{4}, 0.2 \mathrm{~g} \mathrm{~L}^{-1} \mathrm{NaHCO}_{3}, 1.1 \mathrm{~g} \mathrm{~L}^{-1} \mathrm{CaCl}_{2}$ and $0.28 \mathrm{~g} \mathrm{~L}^{-1} \mathrm{NaBr}$.

\section{Results and Discussion}

As depicted in Figure 1a, the synthetic procedure for Zif-8 nanosheets array-coated carbon steel includes two stages. Initially, the oxygen-containing groups on the carbon-steel surface promote the coordination of zinc ions and 2-methylimidazole molecules to generate abundant Zif- 8 nuclei on the surface of carbon steel $[15,16]$. As the reaction progresses, the nuclei grow and act as buds for crystal growth to form uniform nanosheets on carbon steel, as revealed by the SEM images (Figure 1b,c). In addition, the thickness of the prepared Zif-8 layer was measured to be $200 \mathrm{~nm}$ (Figure S1). Furthermore, element-mapping images show that Zn, C and N are distributed homogeneously on the Zif- 8 nanosheet (Figure 1d).

Figure 2a shows the XRD pattern of Zif-8 coating film grown on carbon steel. It is clear that all the diffraction peaks match well with the pattern of Zif- 8 (Figure S2) and the related literature [9]. Additionally, two significant peaks from carbon steel at $44.3^{\circ}$ and $64.4^{\circ}$ are observable (Figure S3).

Furthermore, the corresponding FT-IR spectrum demonstrates similar features to those in the previous work [11]. For example, the peak at $423 \mathrm{~cm}^{-1}$ corresponds to the $\mathrm{Zn}-\mathrm{N}$ stretching vibration, while the peaks at around 994 and $1142 \mathrm{~cm}^{-1}$ belong to the $\mathrm{C}-\mathrm{N}$ vibrations, and the peak at $1566 \mathrm{~cm}^{-1}$ represents $C=\mathrm{N}$ vibration. The peaks at 1383 and $1426 \mathrm{~cm}^{-1}$ are resultant of the methyl bending vibrations. The peaks at 3129 and $2916 \mathrm{~cm}^{-1}$ are assigned to $\mathrm{C}-\mathrm{H}$ stretching vibrations. Additionally, the peaks at $600-800 \mathrm{~cm}^{-1}$ correspond to out-of-plane ring bending vibrations. 


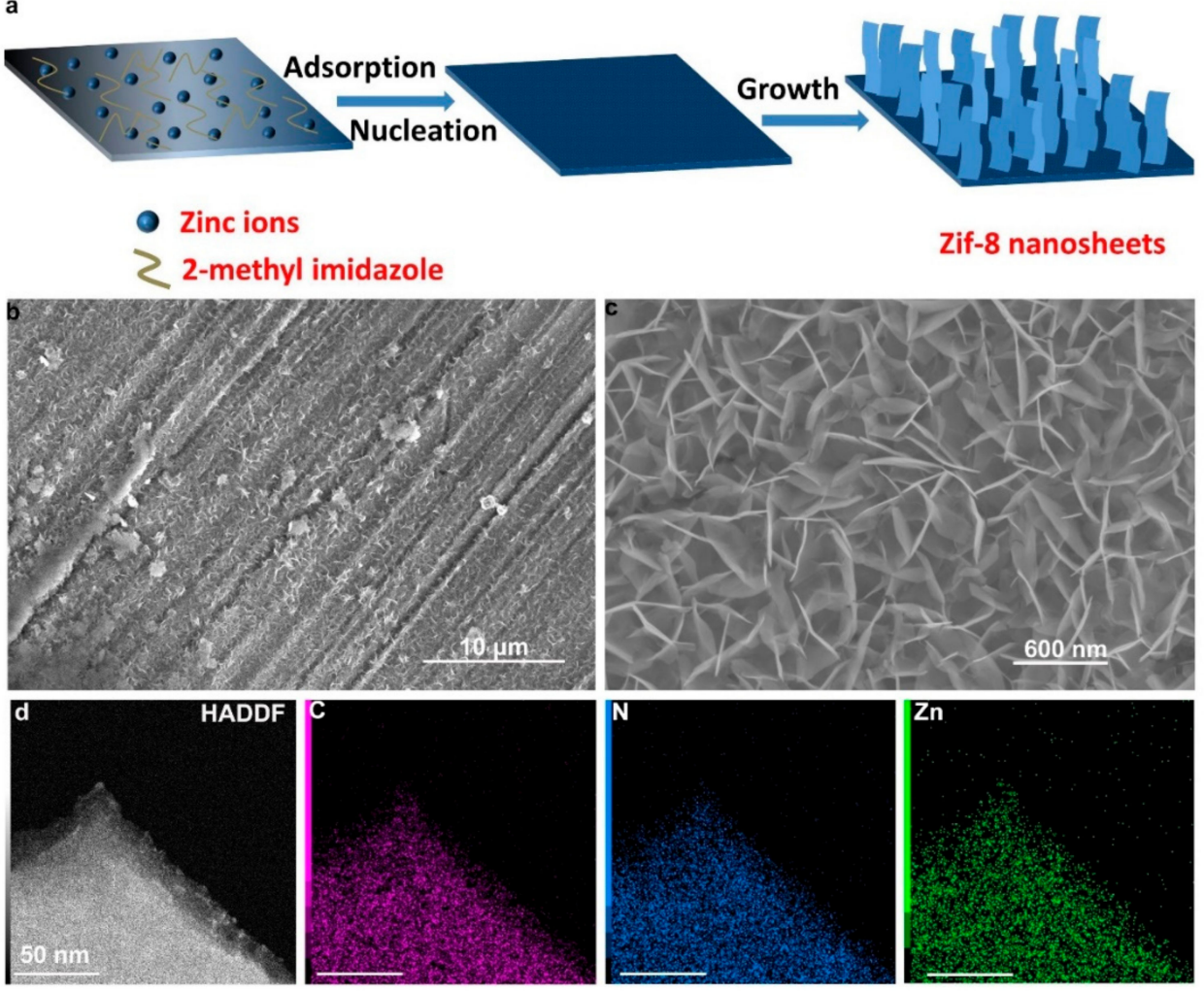

Figure 1. (a) Schematic illustration of the synthesis process. (b,c) SEM images of the Zif- 8 coating sample. (d) STEM image and the corresponding elemental mapping images of Zif- 8 coating sample.
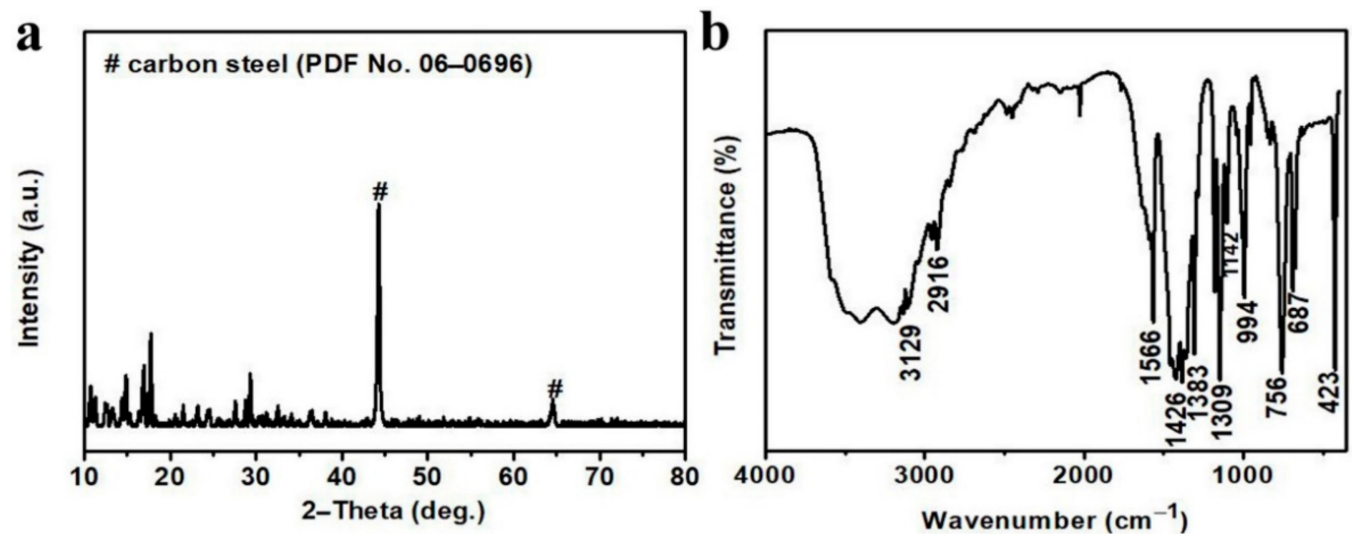

Figure 2. (a) XRD pattern and (b) FT-IR spectrum of the Zif-8 coating sample.

The potentiodynamic polarization curves of the blank and Zif- 8 coating samples are shown in Figure $3 a$. It is well known that a more positive $E_{\text {corr }}$ and a lower $j_{\text {corr }}$ translate to a better anti-corrosion performance [3,4]. As expected, the Zif-8 coating sample exhibits an

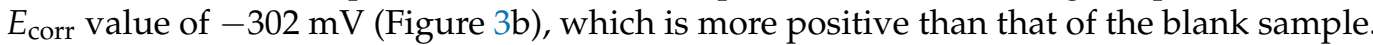
Likewise, the calculated $j_{\text {corr }}$ of the Zif- 8 coating sample is $23 \mu \mathrm{A} \cdot \mathrm{cm}^{-2}$ (Figure $3 \mathrm{~b}$ ), which is obviously smaller than that of the blank sample $\left(225 \mu \mathrm{A} \cdot \mathrm{cm}^{-2}\right)$. The two values confirm that the introduction of the Zif- 8 coating film greatly decreases the corrosion rate of carbon steel in seawater.

Nyquist plots of the blank and Zif-8 coating samples are presented in Figure 3c. A fitting routine was adopted using an equivalent circuit model representing carbon steel substrate, both with and without Zif- 8 coating (see inset of Figure $3 c$ and Table S1). It can be seen that the ZIF- 8 coating sample shows a larger $R_{p}$ of $4010 \Omega \cdot \mathrm{cm}^{-2}$ (Figure $3 b$ ) in comparison to the blank one $\left(2235 \Omega \cdot \mathrm{cm}^{-2}\right)$. This result further suggests that the presence 
of the Zif-8 coating film can effectively hinder the electron transfer from anodic to cathodic sites, thus disrupting the corrosion process [17].
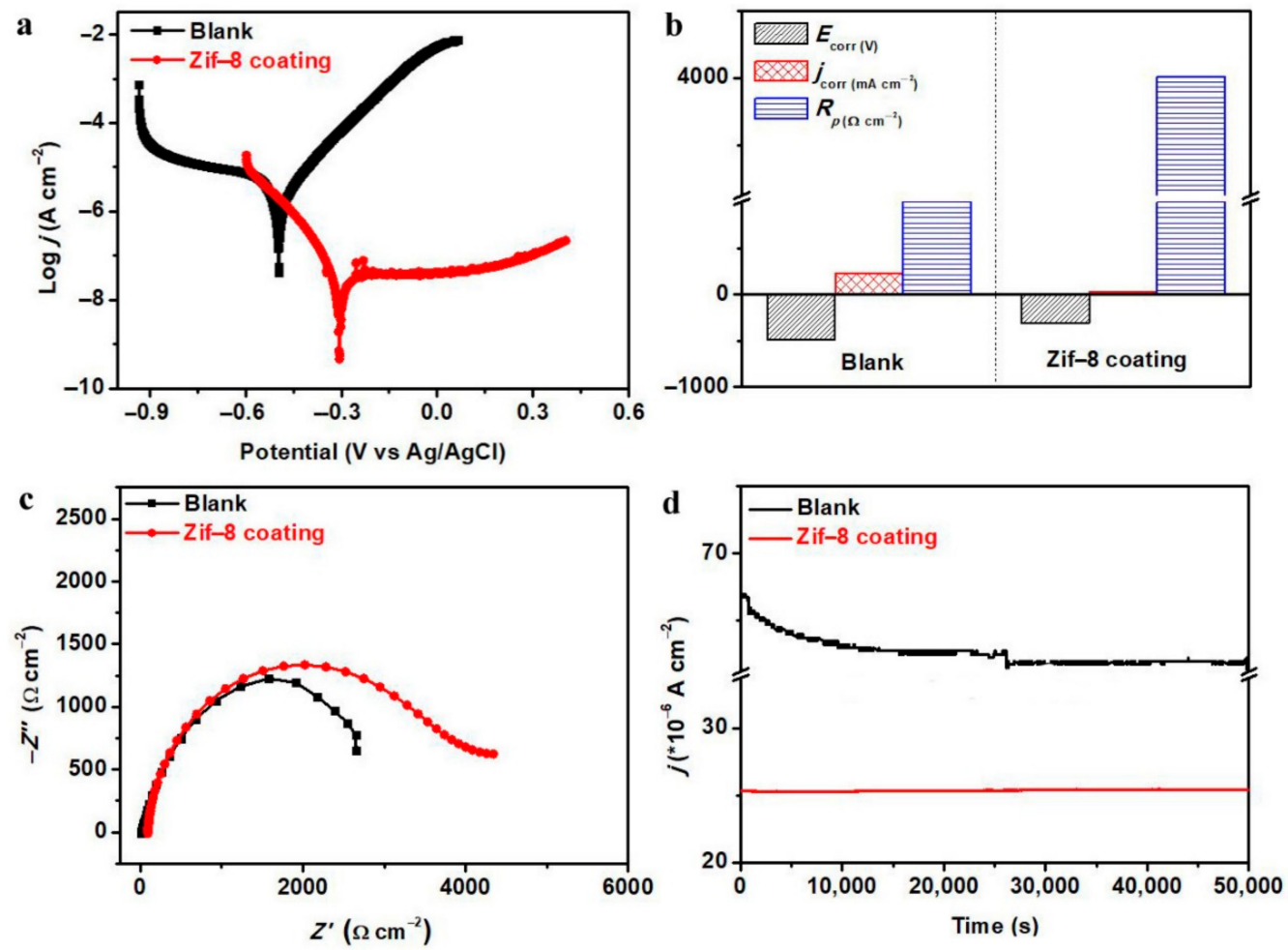

Figure 3. (a) Polarization curves. $j$ denotes the current density. (b) The calculated polarization potential $\left(E_{\text {corr }}\right)$, corrosion current density $\left(j_{\text {corr }}\right)$ and polarization resistance $\left(R_{\mathrm{p}}\right)$. (c) EIS spectra and the corresponding equivalent circuit. (d) Potentiostatic polarization curves for Zif- 8 coating sample at open circuit potential. Note that the electrochemical tests were conducted in artificial seawater.

Furthermore, potentiostatic polarization curves of the blank and Zif- 8 coating samples in seawater were recorded, as shown in Figure 3d. It can be seen that the Zif- 8 coating sample has a smaller passive current density compared to the blank sample, which is in accordance with the result of the potentiodynamic polarization curve.

Additionally, the morphological changes in the blank and Zif- 8 coating samples after immersion in seawater for 15 days were observed by optical images (Figures S4 and S5). As expected, the carbon-steel surfaces are severely corroded due to the reaction of Fe and halide ions [10,18-20]. By contrast, no obvious rust was observed for the Zif- 8 coating sample. Most importantly, the corrosion resistance is still well preserved (Figure S6). Besides, SEM images further confirm that both the integrity of the Zif- 8 coating film and the morphology of nanosheets array are well maintained (Figures S7 and S8).

\section{Conclusions}

In summary, this paper presents the in-situ synthesis of a Zif- 8 nanosheets array on carbon steel with a thickness of $\sim 200 \mathrm{~nm}$, which exhibits efficient anti-corrosion performance. In artificial seawater, the Zif-8-coated sample shows high corrosion resistance $\left(4010 \Omega \cdot \mathrm{cm}^{-2}\right)$ and low corrosion current density $\left(23 \mu \mathrm{A} \cdot \mathrm{cm}^{-2}\right)$ compared to the uncoated sample, which implies higher anti-corrosion properties. Moreover, after immersion in artificial seawater for 15 days, the integrity of the Zif- 8 coating film and the nanosheet-like morphology were well maintained. This work may facilitate the development of MOFsbased coatings for anti-corrosion applications on carbon steel and other materials. The future research on MOFs for corrosion protection will focus on the exploration of their corrosion-prevention mechanism, particularly in seawater. 


\begin{abstract}
Supplementary Materials: The following supporting information can be downloaded at: https:/ / www.mdpi.com/article/10.3390/coatings12030318/s1, Figure S1: Cross sectional SEM images of the uncoated carbon steel (a) and Zif- 8 coating sample (b) before immersed in seawater, Figure S2: XRD patterns of the Zif- 8 powder (red curve) and Zif- 8 coating sample (black curve). As seen, the peaks from $10^{\circ}$ to $40^{\circ}$ can be ascribed to the Zif- 8 phase (JPCDS \#062-1030), Figure S3: XRD pattern of the carbon steel support, Figure S4: Photographs of the blank sample in seawater with different days, Figure S5: Photographs of the Zif-8 coating sample in seawater with different days, Figure S6: EIS spectra before and after long-term immersion in seawater, Figure S7: SEM images of the Zif- 8 coating sample immersed in seawater with 7 days (a) and 15 days (b), Figure S8: Cross sectional SEM image of the Zif- 8 coating sample after immersed in seawater for 15 days. we speculate that some Zif- 8 nanosheets seemed to fall away from the carbon steel during the long-term immersion test and then covered on the surface of Zif- 8 coating film, Table S1: The value of equivalent circuits for curve fitting of uncoated and Zif- 8 coating samples.
\end{abstract}

Author Contributions: Conceptualization, Z.Z. and Q.L.; formal analysis, G.Q.; data curation, Z.Z.; writing-original draft preparation, Q.L.; writing—review and editing, G.Q. All authors have read and agreed to the published version of the manuscript.

Funding: This research was funded by the TJUT Horizontal Topic Cooperation Projects (70304803), the Project of Tianjin Science and Technology, China (20YDTPJC01730) and the Project of China National Offshore Oil Corporation, China (ZX2021ZCZBF6005).

Institutional Review Board Statement: Not applicable.

Informed Consent Statement: Not applicable.

Data Availability Statement: Not applicable.

Conflicts of Interest: The authors declare no conflict of interest.

\title{
References
}

1. Zheng, Y.F.; Gu, X.N.; Witte, F. Biodegradable metals. Mater. Sci. Eng. R Rep. 2014, 77, 1-34. [CrossRef]

2. Cheng, J.; Liu, B.; Wu, Y.H.; Zheng, Y.F. Comparative invitro Study on Pure Metals (Fe, Mn, Mg, Zn and W) as Biodegradable Metals. J. Mater. Sci. Technol. 2013, 29, 619-627. [CrossRef]

3. Yu, L.; Zhao, Z.; Tang, C.; Li, W.; You, C.; Chen, M. The mechanical and corrosion resistance of Mg-Zn-Ca-Ag alloys: The influence of Ag content. J. Mater. Res. Technol. 2020, 9, 10863-10875. [CrossRef]

4. Jiang, S.; Zhang, Z.; Wang, D.; Wen, Y.; Peng, N.; Shang, W. Zif-8-based micro-arc oxidation composite coatings enhanced the corrosion resistance and superhydrophobicity of a $\mathrm{Mg}$ alloy. J. Magnes. Alloy. 2022, in press. [CrossRef]

5. Li, Y.; Chen, M.; Li, W.; Wang, Q.; Wang, Y.; You, C. Preparation, characteristics and corrosion properties of $\alpha$-Al2O3 coatings on 10B21 carbon steel by micro-arc oxidation. Surf. Coat. Technol. 2019, 358, 637-645. [CrossRef]

6. Tian, Y.; Dong, C.; Wang, G.; Cheng, X.; Li, X. Zn-Al-NO 2 layered double hydroxide as a controlled-release corrosion inhibitor for steel reinforcements. Mater. Lett. 2019, 236, 517-520. [CrossRef]

7. Cui, L.-Y.; Zeng, R.-C.; Li, S.-Q.; Zhang, F.; Han, E.-H. Corrosion resistance of layer-by-layer assembled polyvinylpyrrolidone/polyacrylic acid and amorphous silica films on AZ31 magnesium alloys. RSC Adv. 2016, 6, 63107-63116. [CrossRef]

8. Hang, T.T.X.; Truc, T.A.; Duong, N.T.; Pébère, N.; Olivier, M.-G. Layered double hydroxides as containers of inhibitors in organic coatings for corrosion protection of carbon steel. Prog. Org. Coat. 2012, 74, 343-348. [CrossRef]

9. Lü, F.; Zhao, S.; Guo, R.; He, J.; Peng, X.; Bao, H.; Fu, J.; Han, L.; Qi, G.; Luo, J.; et al. Nitrogen-coordinated single Fe sites for efficient electrocatalytic N2 fixation in neutral media. Nano Energy 2019, 61, 420-427. [CrossRef]

10. Zheng, J.; Cui, X.; Yang, Q.; Ren, Q.; Yang, Y.; Xing, H. Shaping of ultrahigh-loading MOF pellet with a strongly anti-tearing binder for gas separation and storage. Chem. Eng. J. 2018, 354, 1075-1082. [CrossRef]

11. Torad, N.L.; Li, Y.; Ishihara, S.; Ariga, K.; Kamachi, Y.; Lian, H.-Y.; Hamoudi, H.; Sakka, Y.; Chaikittisilp, W.; Wu, K.C.W.; et al. MOF-derived Nanoporous Carbon as Intracellular Drug Delivery Carriers. Chem. Lett. 2014, 43, 717-719. [CrossRef]

12. Zhu, Q.; Zhuang, W.; Chen, Y.; Wang, Z.; Hernandez, B.V.; Wu, J.; Yang, P.; Liu, D.; Zhu, C.; Ying, H.; et al. Nano-Biocatalysts of Cyt c@ZIF-8/GO Composites with High Recyclability via a de Novo Approach. ACS Appl. Mater. Interfaces 2018, 10, 16066-16076. [CrossRef] [PubMed]

13. Yang, M.; Sun, J.; Qin, Y.; Yang, H.; Zhang, S.; Liu, X.; Luo, J. Hollow CoFe-layered double hydroxide polyhedrons for highly efficient $\mathrm{CO}_{2}$ electrolysis. Sci. China Mater. 2022, 65, 536-542. [CrossRef]

14. Jiang, H.; Liu, X.-C.; Wu, Y.; Shu, Y.; Gong, X.; Ke, F.-S.; Deng, H. Metal-Organic Frameworks for High Charge-Discharge Rates in Lithium-Sulfur Batteries. Angew. Chem. Int. Ed. 2018, 57, 3916-3921. [CrossRef] [PubMed]

15. Jiang, L.; Dong, Y.; Yuan, Y.; Zhou, X.; Liu, Y.; Meng, X. Recent advances of metal-organic frameworks in corrosion protection: From synthesis to applications. Chem. Eng. J. 2022, 430, 132823. [CrossRef] 
16. Yu, K.; Tan, X.; Hu, Y.; Chen, F.; Li, S. Microstructure effects on the electrochemical corrosion properties of Mg>-4.1\% Ga-2.2\% $\mathrm{Hg}$ alloy as the anode for seawater-activated batteries. Corros. Sci. 2011, 53, 2035-2040. [CrossRef]

17. Furukawa, S.; Reboul, J.; Diring, S.; Sumida, K.; Kitagawa, S. Structuring of metal-organic frameworks at the mesoscopic/macroscopic scale. Chem. Soc. Rev. 2014, 43, 5700-5734. [CrossRef] [PubMed]

18. Yang, Z.; Cheng, Q.; Li, W.; Li, Y.; Yang, C.; Tao, K.; Han, L. Construction of 2D ZIF-derived hierarchical and hollow NiCo-LDH "nanosheet-on-nanosheet" arrays on reduced graphene oxide/Ni foam for boosted electrochemical energy storage. J. Alloys Compd. 2021, 850, 156864. [CrossRef]

19. Fang, G.; Zhou, J.; Liang, C.; Pan, A.; Zhang, C.; Tang, Y.; Tan, X.; Liu, J.; Liang, S. MOFs nanosheets derived porous metal oxide-coated three-dimensional substrates for lithium-ion battery applications. Nano Energy 2016, 26, 57-65. [CrossRef]

20. Kamil, M.P.; Kim, M.J.; Ko, Y.G. Direct electro-co-deposition of Ni-reduced graphene oxide composite coating for anti-corrosion application. Mater. Lett. 2020, 273, 127911. [CrossRef] 